\title{
Flood hazard mapping techniques with LiDAR in the absence of river bathymetry data
}

\author{
Guénolé Choné ${ }^{1, *}$ Pascale M. Biron ${ }^{1}$, and Thomas Buffin-Bélanger ${ }^{2}$ \\ ${ }^{1}$ Department of Geography, Planning and Environment, Concordia University, 1455 De Maisonneuve \\ Blvd W., Montreal, Quebec, H3G 1M8, Canada \\ ${ }^{2}$ Département de biologie, chimie et géographie, Université du Québec à Rimouski, 300 allée des \\ Ursulines, Rimouski, Quebec, G5L 3A1, Canada
}

\begin{abstract}
In many areas of the world, flood risk assessment is either out of date or completely lacking. In Quebec (Canada), one of the challenges to map flood risk is the very large territory combined with very few datasets on river bathymetry, which are required to run hydraulic models. The objective of this study is to assess the precision and accuracy of $2 \mathrm{D}$ flood hydraulic modelling exclusively based on LiDAR elevation data which do not include information on in-channel river bathymetry. Hydraulic simulations (HEC-RAS 5.0) are carried out, for discharges of $20-, 100$ - and 500-year recurrence intervals, using two techniques that do not require bathymetry data, either subtracting discharge of the LiDAR survey from the flood discharge or estimating flow depth from the water surface slope. These techniques are compared to a traditional approach using bed topography obtained from detailed field surveys, on two long reaches (several kilometers). Sensitivity tests were conducted to assess the impacts of the main sources of error on simulated flood levels. Results show that both techniques can be applied with limited introduction of error in the modelled flood stages, and that errors are greatly reduced if calibration data are available.
\end{abstract}

\section{Introduction}

In a context where extreme events are likely to occur more frequently due to both climate change and enhanced urbanization, it is important that flood managers have both a sound knowledge of flood risk zones and the possibility to revise their lateral and vertical extensions on a regular basis. Recent advances in computational capacity have allowed marked improvements in large-scale hydraulics [1,2] that provide the capability of delineating flood risk zones over large territories and on a frequent basis.

In countries like Canada with a large territory and scarce datasets on bathymetry, running hydraulic models to determine flood levels and extensions remains a challenge [3]. This is particularly the case when the accuracy of the simulated levels must be sufficiently good to be included in urban plans with legal implications for permitted residential development. Typically, the bathymetry is estimated as a rectangular channel with width

\footnotetext{
*Corresponding author: guenole.chone@concordia.ca
} 
and depth obtained from classic hydraulic geometry [4], which can then be used in a relationship between channel width and height of channel banks defined after calibrating with observations [3]. Larger rivers can be "burned" directly into the Digital Elevation Model (DEM) [2].

With the increased availability of high-resolution LiDAR DEMs, it is possible to improve estimates of bathymetry for hydraulic simulations of rivers where no bed topography data are available [5,6]. So far, attempts to estimate bed topography from LiDAR data have been limited to relatively short reaches in shallow braided rivers [5] or in a laboratory flume [6]. There is thus a need to develop methods that are applicable for a variety of river channels over long distances, which can also be easily implemented by consultants using widely known software such as HEC-RAS.

The objectives of this study are 1) to develop methods based exclusively on LiDAR data to simulate flood levels, extents and depths in the absence of bathymetry data for long natural reaches and 2) to assess the precision and accuracy of these methods by comparing simulated levels with hydraulic modelling simulations where bathymetry data are available.

\section{Flood modelling techniques}

Two techniques to model flood without bathymetry data are tested against a traditional flood modelling technique (with bathymetry): the Discharge correction technique (DCT) and the Terrain correction technique (TCT) (Figure 1).

Traditional modelling with bathymetry

1) DEM corrected with bathymetry data acquired during field survey

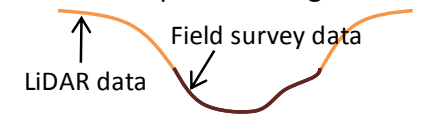

2) 2D flow simulation
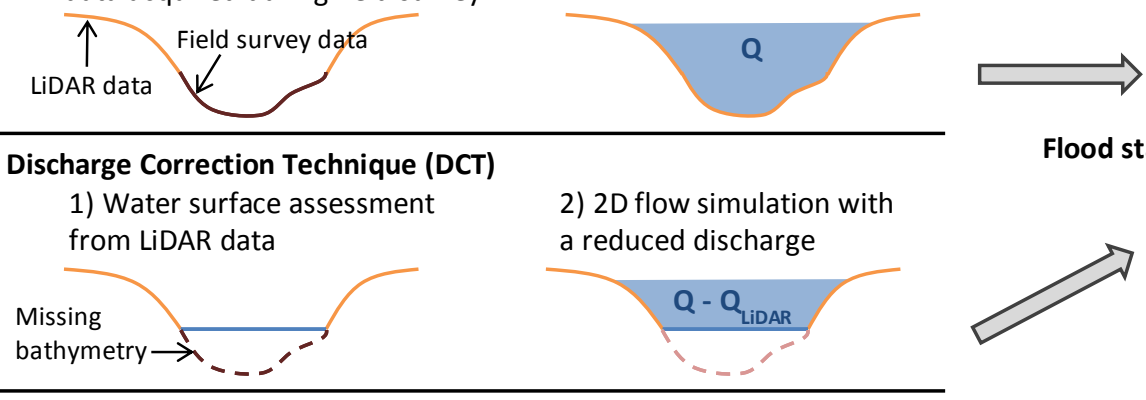

Flood stage comparison

Terrain Correction Technique (TCT)

1) Water surface assessment from LiDAR data

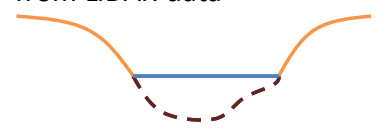

2) 1D flow simulation to estimate

3) 2D flow simulation flow depth and adjust bathymetry on corrected DEM
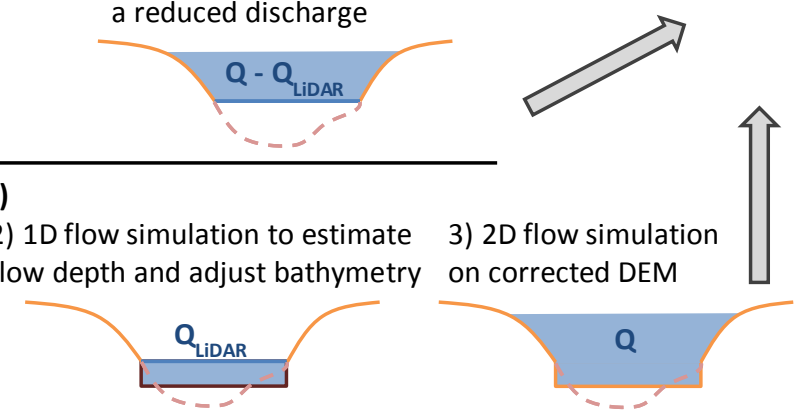

Fig. 1. Flood modelling techniques for a given discharge $\mathrm{Q}$.

The DCT is based on the assumption that a given flow discharge can be separated in two components: the discharge at the time the LiDAR survey took place, below the assessed water surface, and the discharge exceeding the LiDAR discharge, above the assessed water surface. This technique is used commonly to assess discharge of a compound channel, and is also known as the horizontal divided channel method [7]. With this method, the assessed water surface is used as the river bed for flood modelling, but the LiDAR discharge is subtracted from the flood discharge in order to compensate for the missing bathymetry. 
The TCT assesses flow depth when the LiDAR survey took place, and corrects the DEM accordingly. The flow depth is assessed by a 1D flow simulation, at regularly spaced cross-sections. Each cross section is designed as rectangular, with a wetted width measured from the DEM, and the assessed water elevation from the LiDAR acquisition date as the bottom bed elevation. The 1D flow simulation hence uses the water surface slope as a proxy for the bed slope. The resulting calculated flow depth is then subtracted from the original water surface elevation to estimate the true bed elevation, which is burned into the DEM used for flood modelling, using a linear interpolation between cross-sections.

\section{Application of the flood modelling techniques}

\subsection{Study sites}

The traditional model with bathymetry, TCT and DCT were applied on two reaches of two rivers in Quebec, Canada. The first test site is a 1300-m long reach of the Yamaska Sud-Est River (YSE). It is a relatively small meandering river $\left(77 \mathrm{~m}^{3} / \mathrm{s}\right.$ for the 2-year recurrence flood, width of $20 \mathrm{~m}$ approximately), with a slope of 0.0014 , running on the piedmont of the Appalachian range, approximately $90 \mathrm{~km}$ south-east of Montreal. Bed material is mainly sand and gravel. LiDAR data were acquired at relatively low flow $\left(1.5 \mathrm{~m}^{3} / \mathrm{s}, 2 \%\right.$ of the 2 year flood). The second test site is a 3,200-m long reach of the Mastigouche River, situated in the Lanaudière region, $120 \mathrm{~km}$ north-east of Montreal. This meandering river is slightly larger than the YSE $\left(127 \mathrm{~m}^{3} / \mathrm{s}\right.$ for the 2-year recurrence flood, width of $24 \mathrm{~m}$ approximately), with a gentle slope of 0.0004 , a sand bed and clay banks. The LiDAR survey took place during a relative high flow $\left(22.8 \mathrm{~m}^{3} / \mathrm{s}, 18 \%\right.$ of the 2 -year flood).

\subsection{Water surface assessment}

Both DCT and TCT require an assessment of the water surface elevation when the LiDAR data were acquired. The water surface estimated from LiDAR points can be erroneous, either because of the lack of LiDAR points on the water surface [8] or errors in the measured elevation at the water surface [9]. Because the nature of the available data differs at the two study sites, the procedure to determine accurately the water surface from LiDAR was tailored for each site.

LiDAR return intensity can discriminate water bodies [8], and was used on the YSE to filter out potential erroneous LiDAR points. After correcting the point intensity for the incidence angle, as recommended by Höfle and Pfeifer [10], points with an intensity below a threshold were removed before creating a DEM (LiDAR point treatment carried out with Lastools [11] and GIS operations with ArcGIS 10.4). The threshold was assessed manually by observing intensity of sample points in and outside the water surface zone. From remaining LiDAR points, a DEM was generated with a resolution of $20 \mathrm{~m}$ by taking the minimum elevation of LiDAR points inside each raster cell (binning technique), thus allowing to capture the elevation of the point on the bank the closest to the water surface.

On the Mastigouche River, the LiDAR return intensity of the ground points was too low to allow a filtering based on the intensity. The technique designed by Biron et al. [9] was applied in that case.

\subsection{Flood modelling}

TCT and DCT were applied on the study reaches for 20-, 100- and 500-year recurrence floods. Flood discharges were calculated at the closest gauging station on the river with a 
log-Pearson type III statistical analysis, and transferred to the study site using the drainage area ratio approach. Flood simulations were carried out with the 2D model HEC-RAS 5.0.1. The 1D version of HEC-RAS was used for the preliminary assessment of the bed elevation for the TCT. The Manning's n value was set to 0.04 for channel of both rivers, 0.06 for the YSE floodplain, and 0.04 or 0.08 for the Mastigouche River floodplain depending on land use. These values were determined by calibration for the Mastigouche River channel (see section 6), but are arbitrarily set everywhere else.

Models with bathymetry were built using RTK DGPS bed elevation data acquired during field surveys, as well as sonar data for the Mastigouche River. Linear TIN interpolation was used for the YSE between cross-sections (situated roughly every $100 \mathrm{~m}$ ). An anisotropic kriging interpolation was used for the bed in the case of the Mastigouche River. Channel depth obtained by the 1D simulation for the TCT was compared to average depth from the resulting DEM at several cross-sections.

Flood levels resulting from TCT, DCT, and traditional technique (using bathymetry) were first compared using similar Manning's $\mathrm{n}$ for the three techniques in order to assess solely the effect of the modelling technique (section 4) as well as the sensitivity to the main sources of error affecting TCT and DCT (section 5). Using calibration data, and consequently adjusting Manning's n value for each technique, TCT and DCT results were re-assessed (section 6).

\section{Flood modelling techniques comparison}

The flood levels were extracted from the simulated floods at cross-sections with known bathymetry for the YSE ( $\mathrm{n}=13)$, and every $100 \mathrm{~m}$ for the Mastigouche River $(\mathrm{n}=29)$. Flood elevations computed with the DCT and the TCT were then compared to flood elevations computed with the actual bathymetry. Mean error and standard deviation of the error were then used to measure accuracy and precision, respectively, of TCT and DCT (Figure 2). Flood depth was used instead of flood extent to assess the differences between the different models since the presence of valley walls, fluvial terraces or human-made structures results in only small variations in flood extents.

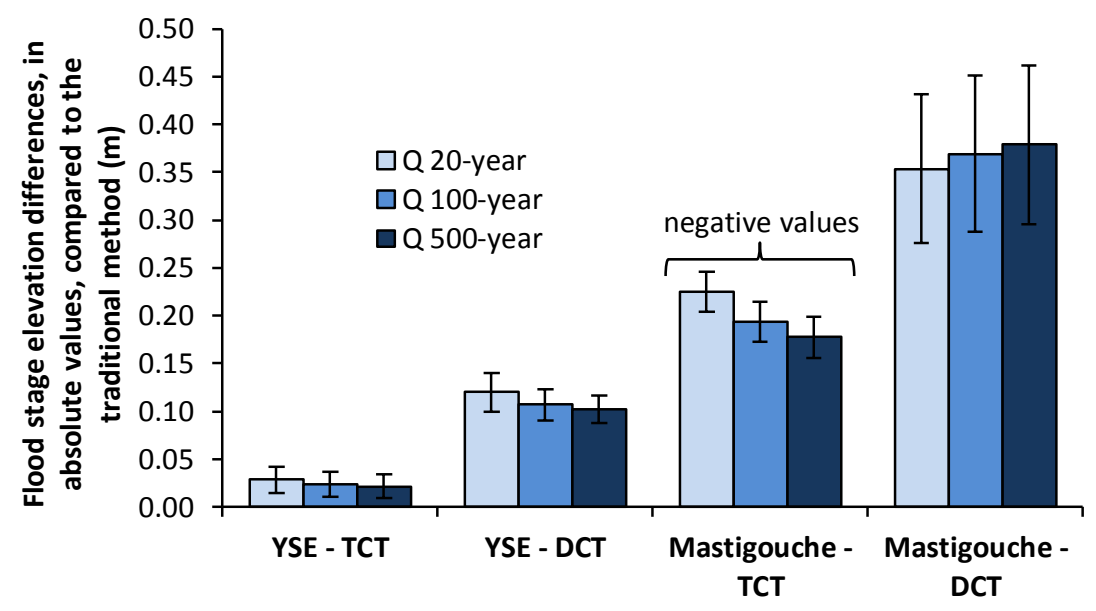

Fig. 2. Mean errors (in absolute values) obtained by comparing simulation results with the TCT or the DCT with traditional simulation results (with bathymetry) for the Yamaska Sud-Est (YSE) and Mastigouche Rivers. Error bars represent the standard deviation of the errors. Note that for the TCT on the Mastigouche River, levels are underestimated (negative mean errors), whereas in all other cases levels are overestimated. 
Four observations are worth noticing from Figure 2. Firstly, TCT and DCT simulate flood levels with high precision, with standard deviations less than $0.02 \mathrm{~m}$ in all cases but the DCT for the Mastigouche River, where values remain low $(0.08 \mathrm{~m})$.

Secondly, TCT results in more accurate flood levels than DCT for all flow discharges. This is not surprising as TCT aims to simulate more accurately the flow stage. The simple assumption in DCT that a given flow in the channel can be divided in two flow components likely underestimates the flow velocity because of the smaller flow depth implied in the modelling of the component above the LIDAR water levels. DCT therefore overestimates flood levels (positive bias) for both rivers. In contrast, TCT results reveal either a positive (YSE) or negative bias (Mastigouche River). Compared to the measured bathymetry, the assessment of the bed elevation for the TCT is relatively good for the YSE $(0.08 \mathrm{~m}$ underestimation), but the bed elevation is markedly underestimated for the Mastigouche River $(0.53 \mathrm{~m})$, leading to an underestimation of the flood stages. This error could come from underestimation of the water stage during the LiDAR survey, overestimation of the inchannel Manning'n, and/or overestimation of the discharge during the LiDAR survey.

Thirdly, errors with both techniques are higher for the Mastigouche River than for the YSE. Several factors could explain this difference. In particular, the gentler slope and the higher relative discharge during the LiDAR survey for the Mastigouche River lead to higher flow depth. A more drastic correction of the bed elevation is therefore needed compared to the YSE, leading to larger absolute elevation errors (Figure 2). In addition, the process used to assess the water elevation during the LiDAR survey for the Mastigouche may not be has accurate as the one used for the YSE due to the lower intensity of the LiDAR signal.

Finally, errors slightly decrease with flood intensity for the TCT on both rivers and the DCT on the YSE, with an opposite trend for the DCT method on the Mastigouche River.

\section{Sensitivity analyses}

\subsection{Sensitivity to discharge during the LiDAR survey}

A possible explanation for the higher error with both TCT and DCT on the Mastigouche River compared to the YSE is that the discharge during the LiDAR survey was $18 \%$ of the 2-year flood, compared to a relatively lower LiDAR discharge on the YSE $(2 \%$ of the 2year flood). The first sensitivity analysis is carried out with the 1D HEC RAS model, with known bathymetry, with different discharge $\left(5,10,20,50\right.$ and $77 \mathrm{~m}^{3} / \mathrm{s}$, corresponding to $7 \%, 13 \%, 26 \%, 65 \%$, and $100 \%$ of the 2 -year recurrence discharge). The resulting simulated water stage is then used as if it were the assessed water stage used to apply the TCT and DCT.

Results reveal that the discharge during the LiDAR survey has a very limited impact on the simulated flood elevation for TCT. A slight overestimation of the flood level, ranging between 0.02 and $0.04 \mathrm{~m}$, with a precision of $0.04 \mathrm{~m}$ or less, is observed for a LiDAR discharge of $65 \%$ of the 2 -year discharge (regardless of the simulated discharges, i.e. 20-, 100- or 500-year flood). This error can reach $0.06 \mathrm{~m}$ if the discharge is a 2-year flood, which is an unlikely situation for a LiDAR survey. For the DCT, however, an important overestimation of the flood level occurs (Figure 3). Furthermore, this overestimation increases greatly with an increase of the discharge during the LiDAR survey, although it remains fairly consistent for the three simulated flood discharges. Consequently, it is possible that, in the case of the DCT simulations on the Mastigouche River, the relatively high discharge during the LiDAR survey contributed to the larger error (Figure 2). 


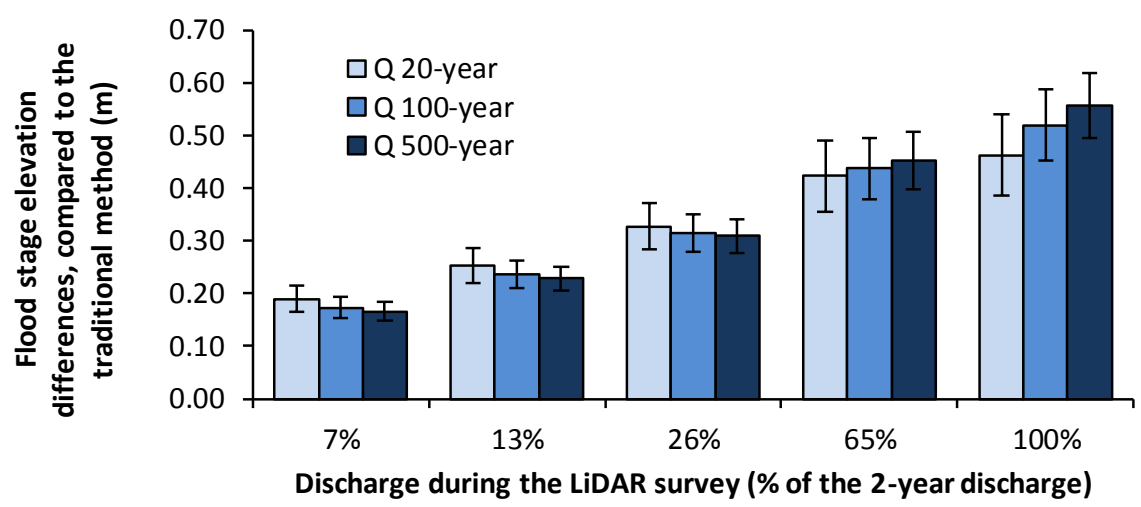

Fig. 3. DCT sensitivity to discharge during LiDAR survey (errors in $\mathrm{m}$, compared to the traditional simulations (with bathymetry) for the YSE. Error bars represent the standard deviation of the errors.

In addition, for LiDAR discharges of $26 \%$ or less than the 2 -year discharge, errors with DCT slightly decrease with the simulated flood discharge (Figure 3). However, for the two highest LiDAR discharges $(65 \%$ and $100 \%$ of the 2 -year value), the opposite trend is observed. While not reaching $26 \%$ of the 2 -year discharge, the nevertheless relatively high LiDAR discharge on the Mastigouche River could be responsible for the observed increase in error with the flood discharge for the DCT (Figure 2).

\subsection{Sensitivity to error in water stage assessment}

This sensitivity analysis was conducted by adding and subtracting $0.20 \mathrm{~m}$ to the originally assessed water elevation during the LiDAR survey. Results reveal that TCT and DCT have similar low sensitivity to this source of error. With both techniques, a $0.20 \mathrm{~m}$ offset in the water elevation induces a 0.06 or $0.07 \mathrm{~m}$ offset in the resulting flood elevation (depending on the flood discharge). Tests with errors ranging between -0.40 and $+0.40 \mathrm{~m}$ confirm the low sensitivity of simulated levels to this variable.

\subsection{Sensitivity to in-channel Manning's $n$}

The roughness of the bed, translated into a Manning's $\mathrm{n}$ value, is often assessed from field observations. Since the objective of the TCT and DCT is to avoid costly field work, accurately assessing Manning's $\mathrm{n}$ remains a challenge. The sensitivity to this parameter of the tested models was assessed by comparing simulated flood elevations using Manning's $\mathrm{n}$ value of 0.06 to those using a value of 0.04 in the channel (Table 1). The three first rows in Table 1 highlight the importance of a good assessment of Manning's $n$ value in any kind of flood simulation, whether or not bathymetry data are available. Indeed, row 1 in Table 1 reveals a difference of $0.30 \mathrm{~m}$ when comparing traditional models with bathymetry using 0.06 versus 0.04 as Manning's n. The errors for TCT and DCT are very similar (rows 2 and 3 , Table 1).

The last two rows of Table 1 highlight an interaction between the discharge during the LiDAR survey and the sensitivity to Manning's n. At a relatively high LiDAR discharge ( $65 \%$ of the 2-year flood), the sensitivity to Manning's $n$ decreases compared to similar results at low LiDAR discharge. This is the case for TCT in particular but also to a lesser degree with DCT. It is noticeable that the sensitivity to an error in Manning's $n$ has a greater impact on the flood elevation with the traditional method (with bathymetry) than with the TCT or DCT. 
Table 1. Sensitivity to Manning's $\mathrm{n}$ within the channel (difference of simulated flood elevation, in $\mathrm{m}$, with $\mathrm{n}=0.06$ compared a similar model using $\mathrm{n}=0.04$ )

\begin{tabular}{|c|c|c|c|}
\hline \multirow{2}{*}{ Model used } & \multicolumn{3}{|c|}{ Simulated flood discharge } \\
\cline { 2 - 4 } & 20 years & 100 years & 500 years \\
\hline Traditional model (with bathymetry) & 0.30 & 0.30 & 0.31 \\
\hline TCT (with real LiDAR discharge) & 0.27 & 0.27 & 0.29 \\
\hline DCT (with real LiDAR discharge) & 0.27 & 0.28 & 0.29 \\
\hline $\begin{array}{c}\text { TCT (with a LiDAR discharge of 65\% } \\
\text { the 2-year flood) }\end{array}$ & 0.11 & 0.12 & 0.14 \\
\hline $\begin{array}{c}\text { DCT (with a LiDAR discharge of 65\% } \\
\text { the 2-year flood) }\end{array}$ & 0.16 & 0.18 & 0.20 \\
\hline
\end{tabular}

\section{Comparison with calibration data}

All previous sensitivity tests on the level of error of different flood modelling techniques were conducted, without calibration data. However, in most flood mapping processes, calibration data are available in the form of flood elevations at certain points, or a map of a flooded area, for a specific flood event. Such data were available in the case of the Mastigouche River for an event that occurred on 20 April 2012 (slightly below a 2-year discharge). These calibration data are used to modify Manning's $n$ values for the three models. The modified Manning's $n$ values of $0.033,0.04$ and 0.021 were then used with the traditional model (with bathymetry), TCT and DCT, respectively (residual error at the calibration point of $0.02 \mathrm{~m}$ or less in the three cases). Error in resulting flood simulations with the TCT and DCT, compared with the traditional model with bathymetry, are presented in Figure 4.

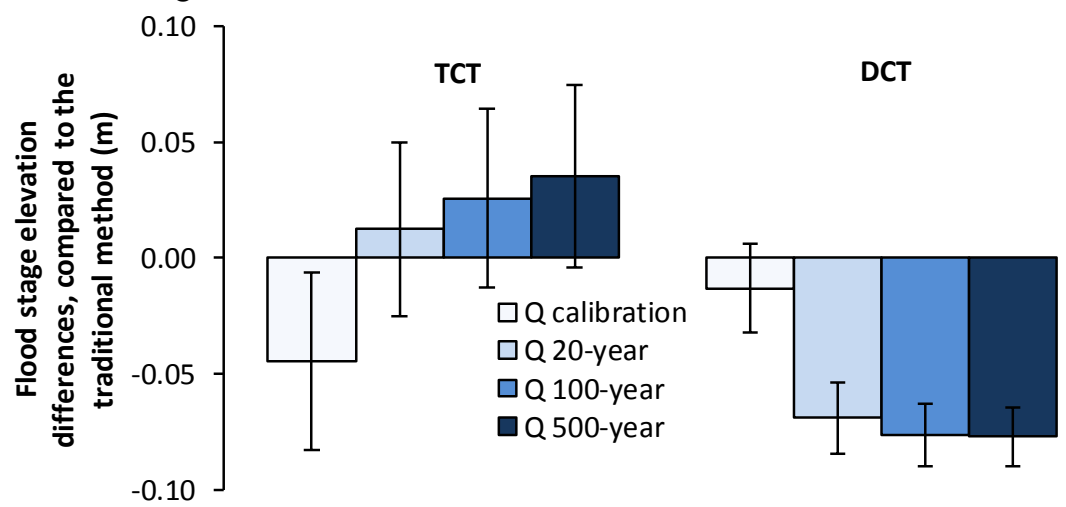

Fig 4. Average differences in simulated flood elevations (in $\mathrm{m}$ ), compared to the traditional model (with bathymetry) after calibration for the Mastigouche River. Error bars represent the standard deviation of the errors.

Considering that an initial mean calibration error of -0.04 and $-0.01 \mathrm{~m}$ occurs with the TCT and DCT, respectively, between these models and the traditional model with the calibration discharge, the resulting simulated flood appear to be overestimated by $0.05,0.07$ and $0.08 \mathrm{~m}$ with the TCT, and underestimated by $0.06,0.07$ and $0.07 \mathrm{~m}$ with the DCT, for the 20-, 100- and 500-years floods respectively. Standard deviations of the errors are less than $0.04 \mathrm{~m}$ and $0.02 \mathrm{~m}$ for the TCT and DCT, respectively. This means that if calibration 
data are available, flood levels can be estimated by both TCT and DCT with a high level of precision and accuracy.

\section{Conclusion}

In the absence of calibration data, and based only on LiDAR data, it is possible to obtain flood level estimates with good precision and reasonable accuracy with the two techniques proposed here: TCT and DCT. The error is, however, larger in the case of a shallow slope channel (Mastigouche River). The TCT, where bathymetry is estimated using a 1D model, provides more accurate results, and is thus recommended, particularly for cases where the LiDAR data were collected at relatively high discharge. In these cases, TCT is also less sensitive to Manning's $n$ than DCT, which subtracts the LiDAR discharge from the flood discharge. However, since DCT always overestimates flood levels (positive bias) compared to traditional simulations with actual bathymetry it may be preferable in cases where a conservative approach is sought, as it is clearly best to overestimate than underestimate flood zones. It is also easier and faster to apply DCT than TCT.

If calibration data are available, both TCT and DCT results in similar flood levels, with a small error (less than $0.08 \mathrm{~m}$ ) compared to runs with bathymetry. Bathymetry data thus provide little information in these cases, meaning that it is possible to avoid costly field campaigns to acquire bathymetry data over long reaches. For areas where flood maps still need to be produced over extensive parts of the territory, as is the case in Quebec, it may thus be more optimal to provide financial resources to acquire high-quality data on water levels during flood events, also making sure that discharge estimates are as accurate as possible by adding gauging stations, than to conduct expensive bathymetry surveys.

The research was supported by the Cadre pour la prévention de sinistres 2013-2020 from the Québec Government [CPS 17-18-01] and by NSERC Discovery grants of T. Buffin-Bélanger and P. Biron.

\section{References}

1. C.C. Sampson, A.M. Smith, P.D. Bates, J.C. Neal, L. Alfieri, J.E. Freer, Water Resour. Res. 51, 7358-7381 (2015)

2. O.E.J. Wing, P.D. Bates, C.C. Sampson, A.M. Smith, K.A. Johnson, T.A. Erikson, Water Resour. Res. 53, 7968-1986 (2017)

3. J. Neal, G. Schumann, P. Bates, Water Resour. Res. 48, W11506 (2012)

4. L.B. Leopold, T.J. Maddock, U.S. Geol. Surv. Prof. Pap. 252 (1953)

5. G.M. Smart, J. Bind, M.J. Duncan, 18th World IMACS / MODSIM Congress, Cairns, Australia, 2521-2527 (2009)

6. A.F. Gessese, M. Sellier, E. Van Houten, G. Smart, $34^{\text {th }}$ IAHR World Congress, Brisbane, Australia, 3466-3472 (2011)

7. K. Bradbrook, S.N. Lane, S.G. Waller, P.D. Bates, Int. J. River Basin Manag. 2, 211223 (2004)

8. B. Höfle, M. Vetter, N. Pfeifer, G. Mandlburger, J. Stötter, Earth Surf. Process. Landf. 34, 1635-1649 (2009)

9. P.M. Biron, G. Choné, T. Buffin-Bélanger, S. Demers, T. Olsen, Earth Surf. Process. Landf. 38, 1808-1821 (2013)

10. B. Höfle, N. Pfeifer, J. Photogramm. Remote Sens. 62, 415-433 (2007)

11. Rapidlasso GmbH. https://rapidlasso.com/lastools/ 